\title{
GLUCOSE EXCRETION IN BRIGHT'S DISEASE ${ }^{1}$
}

By JAMES A. HAWKINS, EATON M. MACKAY AND DONALD D. VAN SLYKE. (From the Hospital of the Rockefeller Institute for Medical Research, New York)

(Received for publication May 29, 1929)

The occurrence of hyperglycemia and glycosuria in nephritis has been observed and studied by Neubauer in 1910 and a number of subsequent investigators. A review of this subect has been included in the article on carbohydrate metabolism in nephritis by Linder, Hiller and Van Slyke (1925). They found that hyperglycemia and glycosuria were most frequent in cases with marked deficit of renal function; that nephritics with fasting hyperglycemia when fed glucose showed abnormally high and prolonged blood sugar curves, similar to those in mild diabetes; that nevertheless the respiratory quotients after glucose feeding rose as in normal subjects, indicating a normal combustion of glucose. The abnormality appeared to lie in a retarded transfer of glucose from blood to tissues, rather than in a retarded combustion. The renal threshold of sugar excretion appeared to be low in some cases, as evidenced by the appearance of a positive qualitative Benedict test for reducing substances in the urine.

However, as shown by Eagle (1926-27) and by Van Slyke and Hawkins (1929), normal urine may contain substances equivalent in reducing power to 0.1 per cent or more of glucose; but only about onetenth of the reduction is actually due to sugar capable of fermentation, like glucose. To obtain interpretable data concerning the permeability of nephritic kidneys to glucose it is therefore necessary to base conclusions upon determinations of the fermentable sugar in the urine. The present studies are based on such determinations.

${ }^{1}$ A preliminary report of results was presented by the authors at the 1928 meeting of the American Society of Biological Chemists (Hawkins,MacKay, and Van Slyke, 1928). 


\section{METHODS}

The blood sugar determinations were made by the microgasometric ferricyanide method of Van Slyke and Hawkins (1928) on cutaneous blood obtained by a clean deep prick with a sharp needle. The blood obtained in this way is practically arterial (Lundsgaard and Möller, 1922). We did not determine the fermentable blood sugar, but used "total blood sugar" values, because the non-fermentable material determined in blood by the ferricyanide method has been shown to be a relatively small and constant value (Van Slyke and Hawkins, 1929).

The reducing substances of the urine, fermentable andnon-fermentable, were determined quantitatively by the Van Slyke-Hawkins gasometric methods (1928, 1929). In considering urinary excretions our conclusions have been based entirely on fermentable sugar values, since the unidentified non-fermentable reducing material in urine is relatively so much that in normal subjects it constitutes nearly all of the total reducing material.

Glucose ingestion tests. The subjects of these studies were given no food or fluids for a 12-hour period preceding the determinations. Each subject was given $200 \mathrm{cc}$. of water at 7:00 a.m. Blood was taken at 7:00 and 8:00 a.m., and urine collected for the period from 7:00 to 8:00 a.m. Glucose was given in $200 \mathrm{cc}$. of water at room temperature at 8:00 a.m. All intervals indicated on the charts are measured from this point. Blood spec.mens were taken at $0.25,0.5,0.75,1.0$, $1.25,1.75,2.15,2.75,3.15$ and 3.75 hours. The urine specimens were collected at $0.5,1.0,1.5,2.0,2.5,3.0$, and 4.0 hours. The subject remained in bed throughout the test and received no other fluid, food, or medication during this period. The amount of glucose given was 1 gram for each kilo of ideal weight estimated for the subject's height, age and sex, by figure 1 of McIntosh, Möller, and Van Slyke (1928). The glucose used was Merck's "C.P."

\section{RESULTS}

Blood sugar curves, and curves indicating the amounts of fermentable and non-fermentable reducing substances in the urine were obtained on 3 normal persons and on 19 patients with nephritis. 
The blood and urine curves of the normal subjects are given in figure 1. Figures 2 to 11 show the curves obtained in the different forms and stages of nephritis.

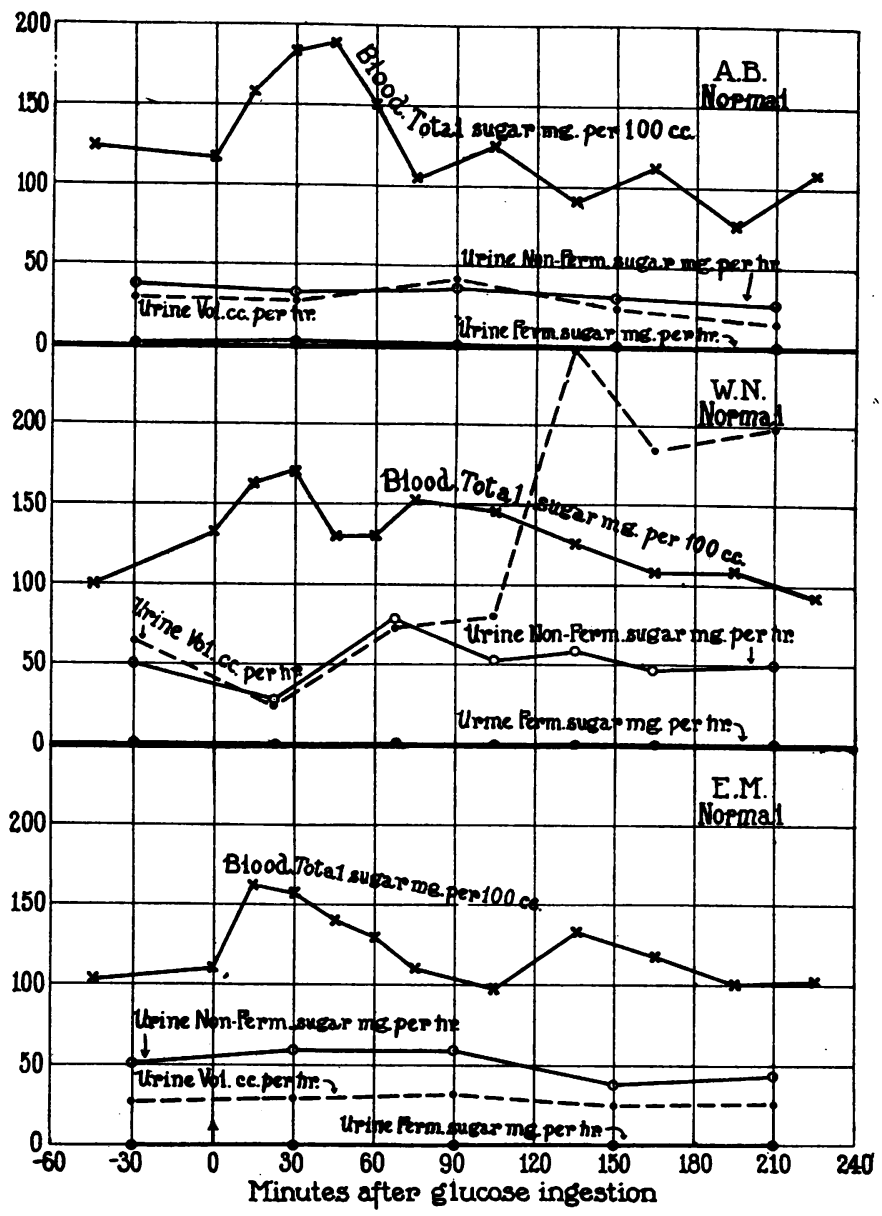

Fig 1. Data on Normal Subjects

In table 1 is given a summary of the tests made on patients with Bright's disease. The Addis classification (1925) for Bright's disease is used, and the patients are grouped according to the divisions and subdivisions of this classification. The age, sex, blood pressure and 
relative extent of edema at the time of the test are reported in Table 1 in order to give some aid in judging the patient's clinical status. The 2-hour phthalein excretion and the urea excreting ability in terms of the blood urea clearance (cc. of blood cleared of urea by 1 minute's excretion determined as described by Möller, McIntosh, and Van Slyke, 1928) are noted to indicate the state of renal function at the time of the tests. The clearance values are given in per cent of average

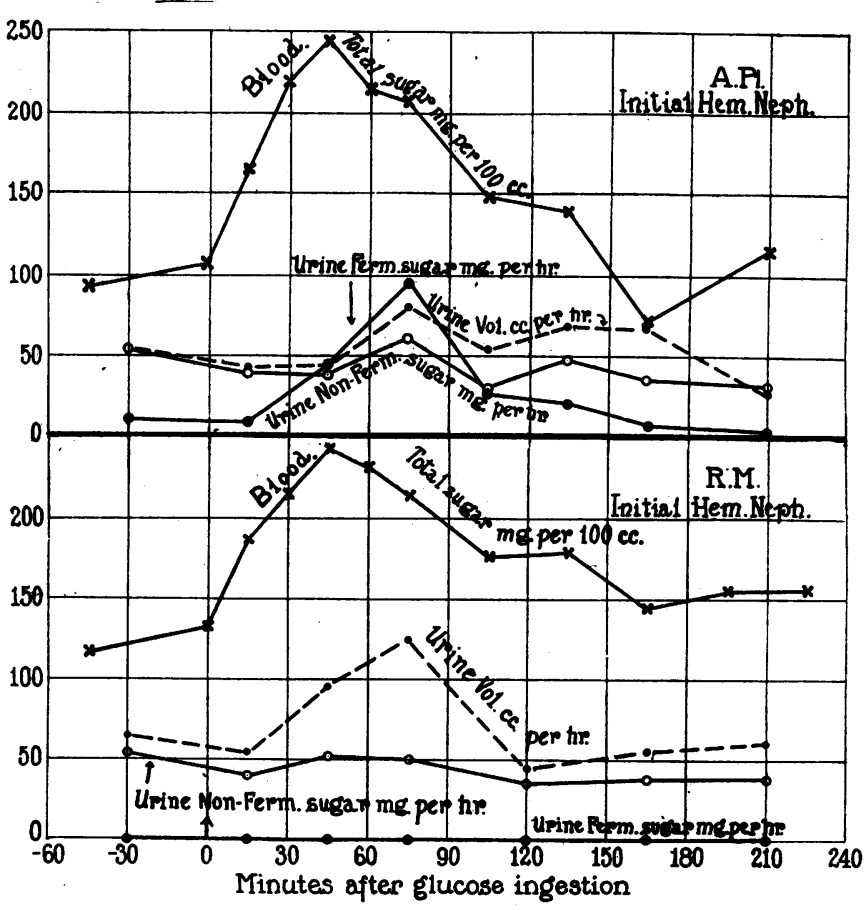

Fig. 2. Data on Subjects with Initial Hemorrhagic Nephritis

normal, viz., $54 \mathrm{cc}$. of blood per minute for the "standard clearance," estimated for urine output of $1 \mathrm{cc}$. per minute; $75 \mathrm{cc}$. per minute for "maximum clearance" with urine volumes exceeding $2 \mathrm{cc}$. per minute.

\section{DISCUSSION}

In normal subjects our data, taken with those of Eagle (1926-27) indicate that excretion of fermentable sugar is usually less than $\mathbf{5} \mathbf{~ m g m}$. 


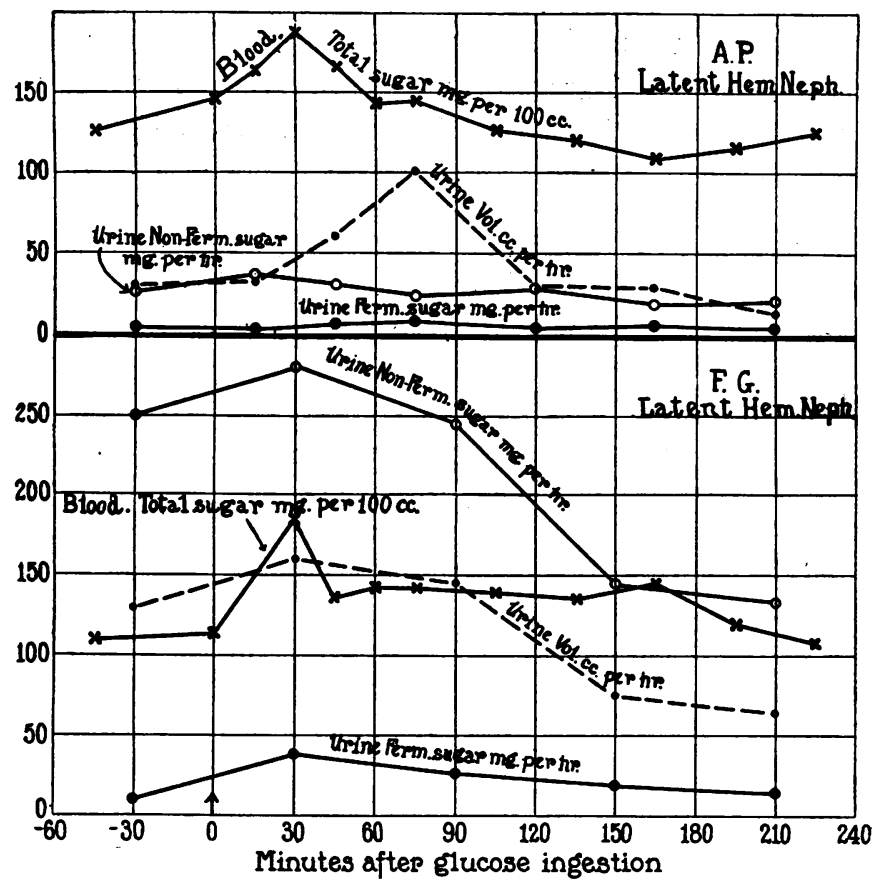

Fig. 3. Data on Subjects with Latent Hemorrhagic Nephritis

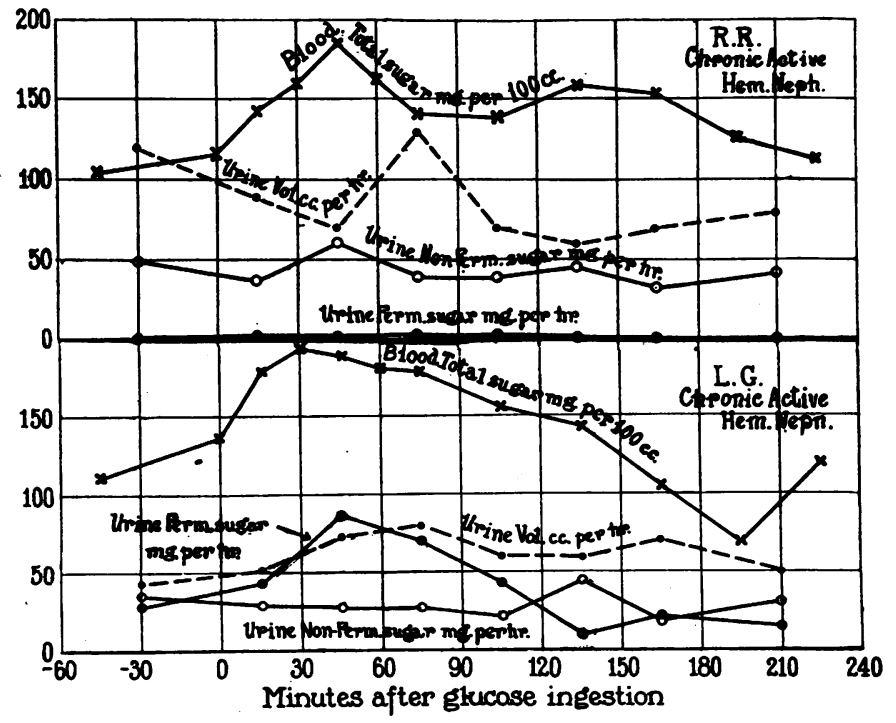

Fig. 4. Data on Subjects with Chronic Active Hemorrhagic Nephritis 
per hour during fast, and is not increased above this level after consumption by mouth of 1 gram of glucose per kilo body weight. It is known, of course, that there are otherwise normal subjects who have

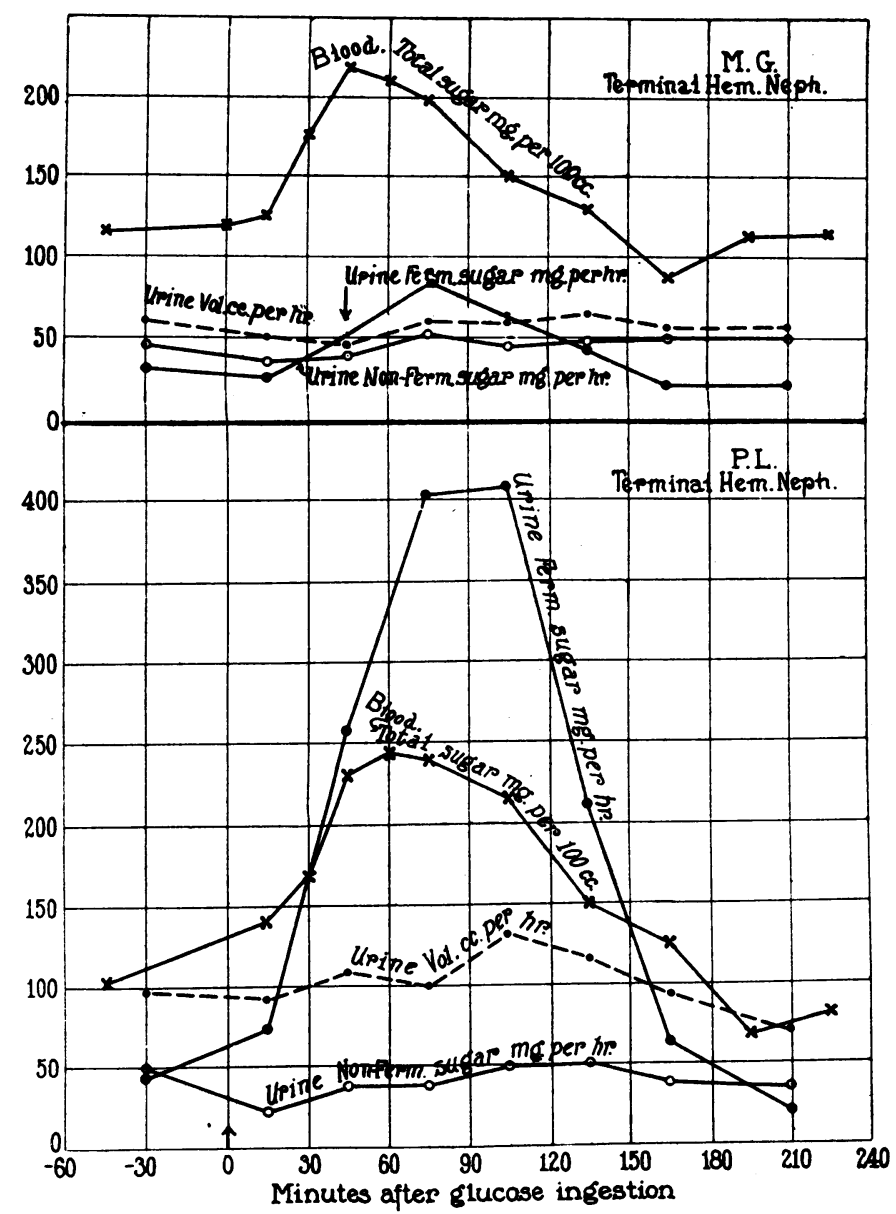

Fig. 5. Data on Subjects with Terminal Hemorrhagic Nephritis

continual or intermittent glycosuria, which appears attributable to a low renal threshold for glucose, since the glycosuria occurs in the presence of normal blood sugar values. Such cases appear, however, to be relatively rare. Our controls and those of Eagle may be taken 
to represent the usual normal behavior, marked departure from which, if frequent in any given condition, may be considered as evidence of deviation from the probable normal behavior of the subjects examined.

Abnormal glycosurias (compared with the above controls) were shown by all our cases with degenerative Bright's disease (nephrosis)

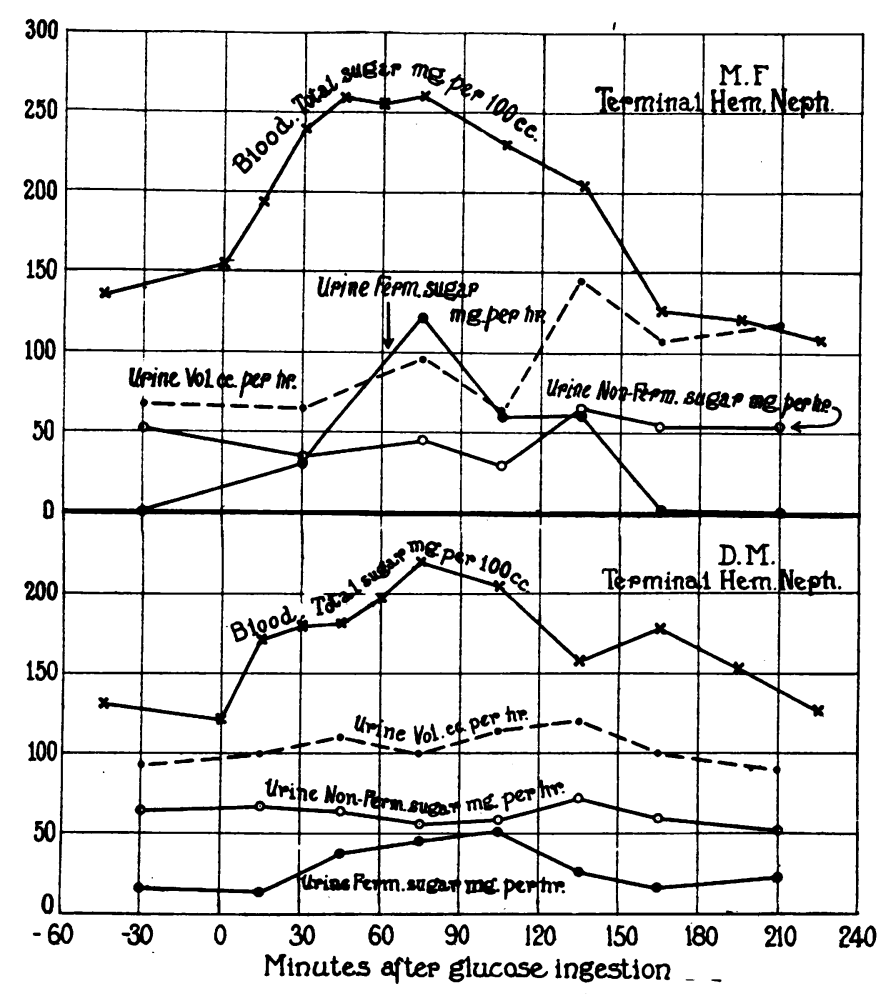

Fig. 6. Data on Subjects with Terminal Hemorrhagic Nephritis

and by all in the terminal stage of hemorrhagic nephritis. In excretion of fermentable sugar after glucose feeding all these patients exceeded the normal controls, and in excretion during fasting all except one, a terminal hemorrhagic, exceeded the controls.

The most marked glycosurias occurred in the degenerative group: 3 out of 6 cases showed over 0.3 per cent of fermentable sugar in the urine during fast, and over 1 per cent after glucose feeding. 
In cases of initial and moderately advanced hemorrhagic nephritis, about half showed relative glycosuria, both fasting and after glucose ingestion.

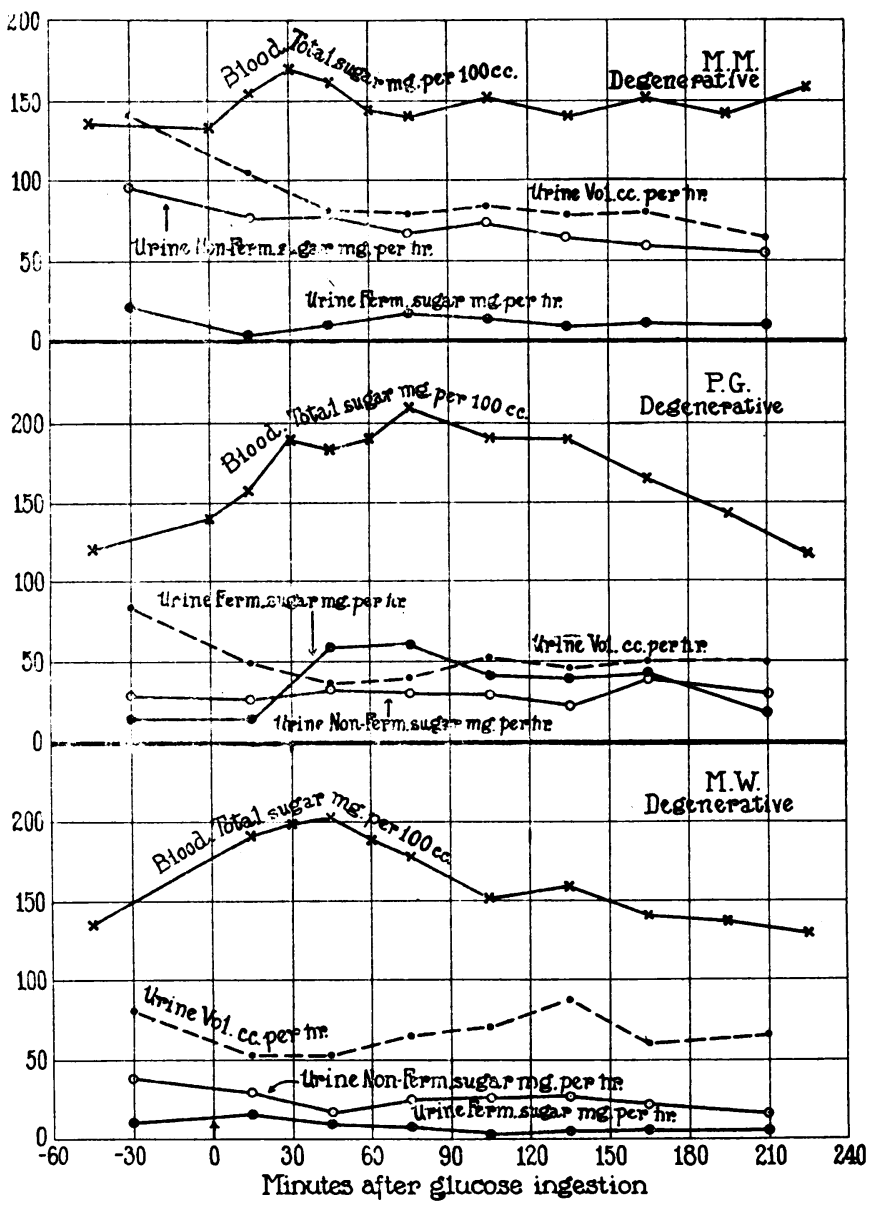

Fig. 7. Data on Subjects with Degenerative Hemorrhagic Nephritis

Of the 3 arteriosclerotic cases one showed a definite glycosuria (50 mgm. per hour) after glucose feeding, none while fasting.

Comparison of blood sugar curves with fermentable sugar excretion indicates that the glycosurias in all types of the disease appear attribut- 
able to low excretion thresholds rather than to high blood sugar values. In some cases, it is true, particularly in the terminal hemorrhagic group, the blood sugar peak after glucose ingestion is so high (210-266 mgm. per cent) that glycosuria would be expected over a normal threshold. However, nearly all such cases showed glycosuria also in the fasting state, with relatively low blood sugar values. The work of Linder, Hiller, and Van Slyke (1925) has shown that somewhat high

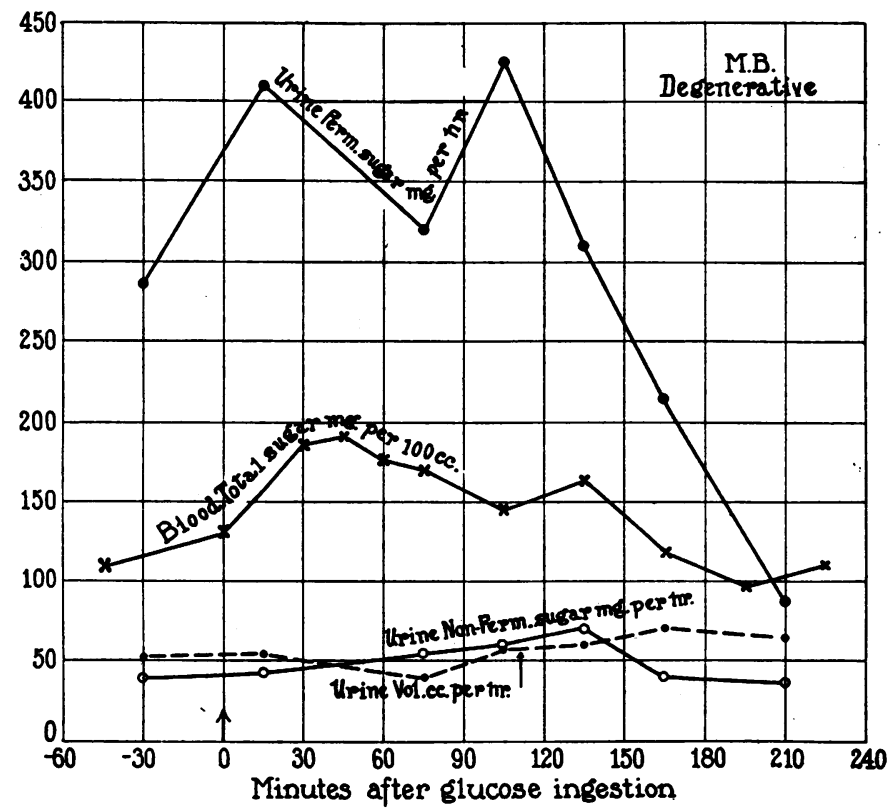

Fig. 8. Data on a Subject with Degenerative Hemorrhagic Nephritis

and prolonged blood sugar curves after glucose feeding are common in nephritis, and are unconnected with any retardation in the rate at which glucose can be burned, or with any other evidence of diabetes.

In the degenerative cases showing gross glycosuria, both fasting and after glucose feeding, the blood sugar curves were within normal limits.

It appears probable that many cases heretofore reported as combined nephritis and diabetes have been nephritis with renal glycosuria. 
It is possible that the glycosuria is due to failure of the renal tubules to reabsorb glucose from the glomerular filtrate. Such an hypothesis

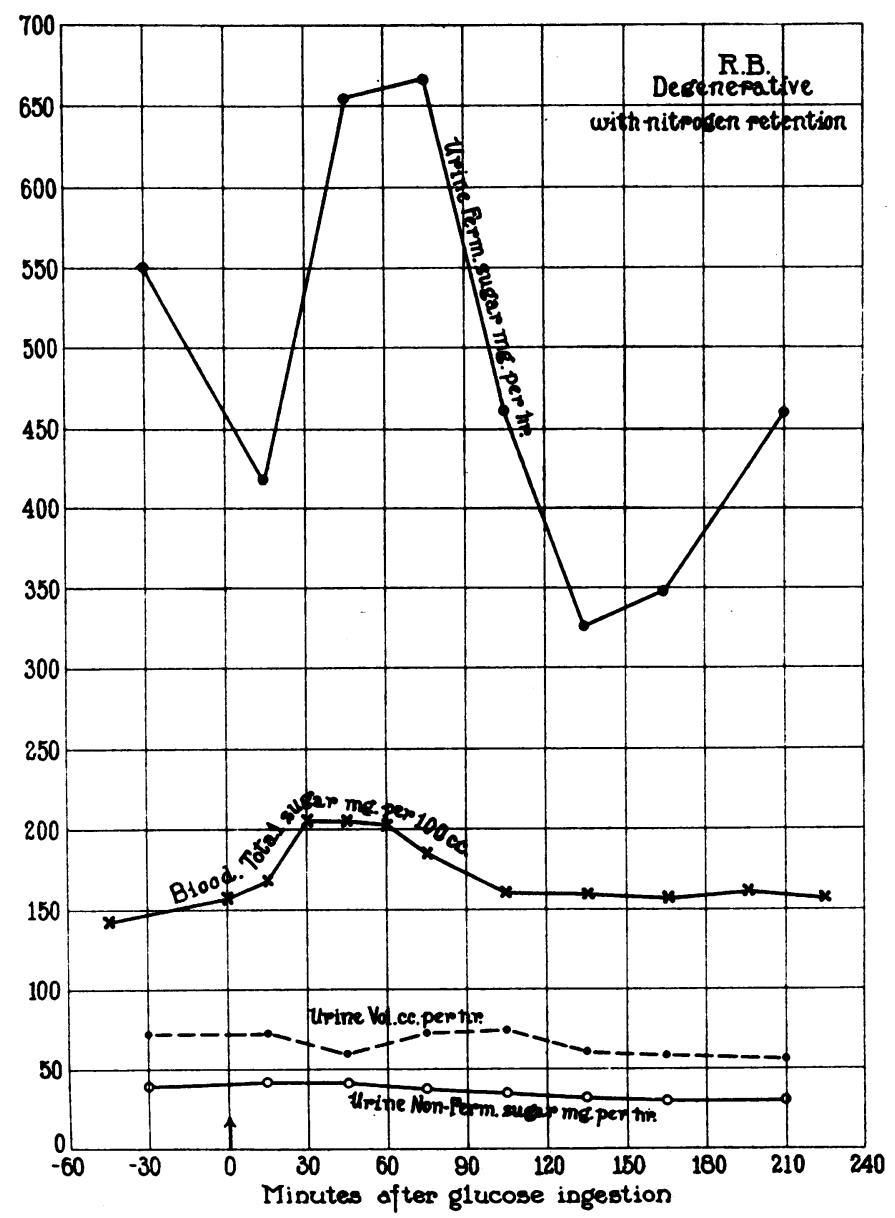

Fig. 9. Data on a Subject, R. B., with Degenerative Hemorrhagic Nephritis With Nitrogen Retention

appears somewhat plausible because of the especially marked glycosurias observed in certain of the degenerative cases, in which the tubules are known to be especially involved. 
J. A. HAWKINS, E. M. MACKAY AND D. D. VAN SLYKE

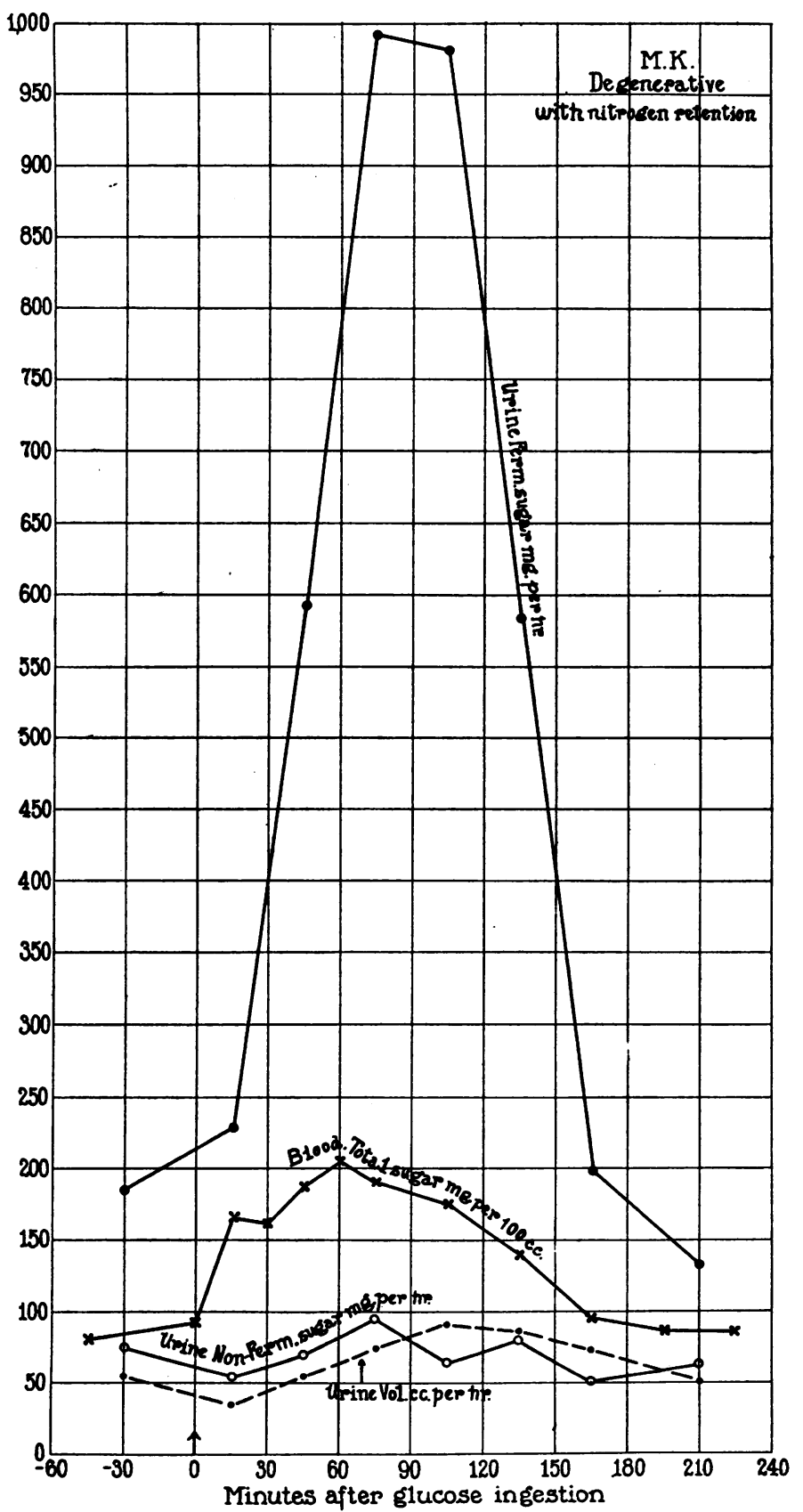

Fig. 10. Data on a Subject, M. K., with Degenertive Hemorrhagic Nephritis With Nitrogen REtention 
TAl

Clinical and labora

\begin{tabular}{|c|c|c|c|c|c|c|c|c|c|c|c|c|c|c|}
\hline \multirow[b]{3}{*}{ 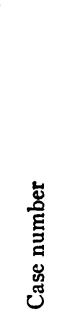 } & \multirow[b]{3}{*}{ 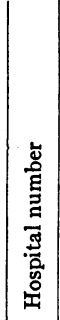 } & \multirow{2}{*}{\multicolumn{2}{|c|}{$\frac{\text { Diagnosis }}{\text { Bright's disease }}$}} & \multirow[b]{3}{*}{ 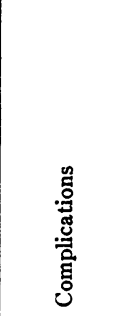 } & \multirow[b]{3}{*}{$\underset{<}{\stackrel{\infty}{<}}$} & \multirow[b]{3}{*}{ ڤั山 } & \multirow[b]{3}{*}{ 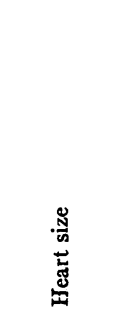 } & \multirow[b]{3}{*}{ 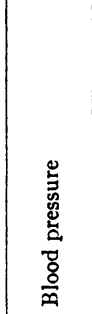 } & \multirow[b]{3}{*}{ 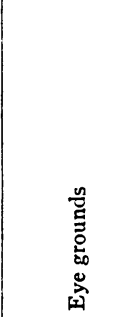 } & \multirow[b]{3}{*}{ 苛 } & \multirow[b]{3}{*}{ 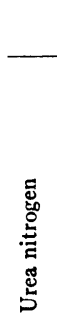 } & \multirow[b]{3}{*}{ 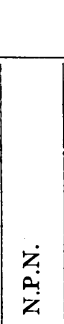 } & & \\
\hline & & & & & & & & & & & & & \multicolumn{2}{|c|}{ Plasma } \\
\hline & & $\stackrel{\mathscr{2}}{\stackrel{\leftrightarrow}{\Leftrightarrow}}$ & $\begin{array}{l}\mathbb{E}_{0} \\
\text { 吾 } \\
\text { w }\end{array}$ & & & & & & & & & & Uू. & c \\
\hline & & & & & $y r s$. & & & & & & $\begin{array}{l}\text { mg. } \\
\text { per } \\
\text { cent }\end{array}$ & $\begin{array}{l}\text { mg. } \\
\text { per } \\
\text { cent }\end{array}$ & $\begin{array}{c}\text { mg. } \\
\text { per } \\
\text { cent }\end{array}$ & $\begin{array}{c}m\} \\
p e \\
\text { cet }\end{array}$ \\
\hline R. M. & 6353 & Hemorrhagic & Initial & & 38 & F: & Normal & $138 / 90$ & Normal & 0 & 15.0 & 35.0 & 1.50 & 34 \\
\hline A. $\mathrm{Pl}$. & 6139 & Hemorrhagic & Initial & & 19 & M. & Normal & $150 / 95$ & Normal & 0 & 23.8 & 48.0 & $|1.97|$ & \\
\hline A. P. & 6251 & Hemorrhagic & Latent & & 13 & M. & Increased & $110 / 60$ & Normal & + & 8.2 & 31.0 & 1.30 & \\
\hline F. G. & 6162 & Hemorrhagic & Latent & . & 20 & F. & $\begin{array}{l}\text { Slightly } \\
\text { increased }\end{array}$ & $200 / 115$ & Normal & 0 & 12.5 & 28.0 & 1.58 & 45 \\
\hline L. G. & 6123 & Hemorrhagic & Active & & 19 & F. & Normal & $150 / 105$ & $\begin{array}{l}\text { Hemor- } \\
\text { rhages }\end{array}$ & + & 30.6 & 51.0 & 2.26 & 81 \\
\hline R. R. & 6475 & Hemorrhagic & Active & & 25 & F. & Normal & $165 / 114$ & Normal & ++ & 8.8 & 27.0 & 1.36 & \\
\hline M. G. & 5074 & Hemorrhagic & Terminal & & 15 & M. & Normal & $150 / 90$ & & 0 & 45.0 & 59.0 & 4.10 & \\
\hline P. L. & 5210 & Hemorrhagic & Terminal & $\begin{array}{l}\text { Chronic } \\
\text { middle } \\
\text { ear }\end{array}$ & 30 & M. & $\begin{array}{l}\text { Slightly } \\
\text { increased }\end{array}$ & $150 / 90$ & & 0 & 78.2 & 118.0 & 4.50 & 22 \\
\hline D. M. & 6238 & Hemorrhagic & Terminal & & 16 & M. & Increased & $135 / 85$ & Normal & 0 & 46.1 & 64.0 & 4.39 & 27 \\
\hline M. F. & 6183 & Hemorrhagic & Terminal & & 30 & F. & Increased & $220 / 150$ & $\begin{array}{l}\text { Hemor- } \\
\text { rhages, } \\
\text { retinitis }\end{array}$ & 0 & 68.4 & 102.0 & 9.00 & \\
\hline P. G. & 6482 & Degenerative & Active & & 25 & M. & Normal & $105 / 75$ & Normal & +++ & 8.8 & 22.0 & 1.36 & 58 \\
\hline M. M. & 6184 & Degenerative & Active & & 18 & M. & Normal & $105 / 75$ & Normal & +++ & 6.6 & 20.0 & 1.33 & 53 \\
\hline M. B. & 6172 & Degenerative & Active & $\begin{array}{l}\text { Pulmonary } \\
\text { tuber- } \\
\text { culosis }\end{array}$ & 29 & M. & Normal & $110 / 70$ & Normal & + & 12.7 & 33.0 & 1.67 & 83 \\
\hline M. W. & 6473 & Degenerative & Active & & 12 & M. & Normal & $115 / 70$ & Normal & +++ & 18.8 & 28.0 & 1.36 & 60 \\
\hline R. B. & 5505 & Degenerative & Terminal & & 11 & M. & Normal & $115 / 60$ & Normal & ++ & 22.3 & 49.0 & 3.16 & \\
\hline M. K. & 5949 & Degenerative & Terminal & Empyema & 20 & M. & Normal & $130 / 80$ & Normal & +++ & 37.3 & 50.0 & 3.10 & 68 \\
\hline K. C. & 6102 & $\begin{array}{l}\text { Arterio- } \\
\text { sclerotic }\end{array}$ & & & 51 & F. & $\begin{array}{l}\text { Slightly } \\
\text { increased }\end{array}$ & $150 / 85$ & Normal & 0 & 15.7 & 36.0 & 1.67 & \\
\hline M. Fe. & 6446 & $\begin{array}{l}\text { Arterio- } \\
\text { sclerotic }\end{array}$ & & & 49 & F. & Normal & $235 / 155$ & Normal & 0 & 11.4 & 14.0 & 1.36 & 31: \\
\hline M. Go. & 6466 & $\begin{array}{l}\text { Arterio- } \\
\text { sclerotic }\end{array}$ & & $\begin{array}{l}\text { Cardiac } \\
\text { failure }\end{array}$ & 49 & F. & $\begin{array}{l}\text { Greatly } \\
\text { increased }\end{array}$ & $240 / 145$ & Retinitis & + & 31.1 & 57.0 & 1.76 & \\
\hline
\end{tabular}


SLE 1

tory data on patients

\begin{tabular}{|c|c|c|c|c|c|c|c|c|c|c|c|c|c|c|c|c|c|c|c|c|c|c|}
\hline \multicolumn{6}{|c|}{ Blood } & \multicolumn{11}{|c|}{ Urine (excretion per 12 hours) } & \multicolumn{2}{|c|}{ Renal function } & \multicolumn{4}{|c|}{ Sugar in blood and urine } \\
\hline & \multicolumn{5}{|c|}{ Plasma proteins } & \multirow[b]{3}{*}{ 足 } & \multicolumn{10}{|c|}{ Formed elements } & \multirow{3}{*}{ 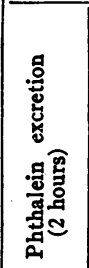 } & \multirow{3}{*}{ 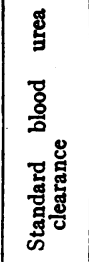 } & \multirow{3}{*}{ 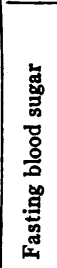 } & \multirow{3}{*}{ 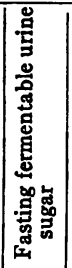 } & \multirow{3}{*}{ 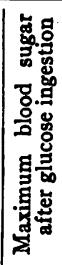 } & \multirow{3}{*}{ 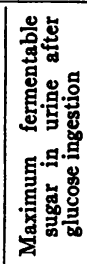 } \\
\hline & & & & & & & & & & & & Cas & & & & & & & & & & \\
\hline ?3 & 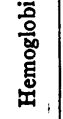 & 虽 & $\begin{array}{l}\text { 章 } \\
\text { 온 }\end{array}$ & 总 & 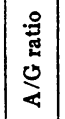 & & 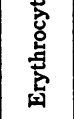 & 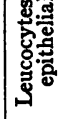 & 胥 & 兽 & $\frac{\overline{8}}{9}$ & . & 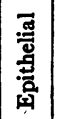 & 总 & 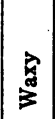 & 莺 & & & & & & \\
\hline $\begin{array}{l}\ddot{r} \\
\text { uे }\end{array}$ & 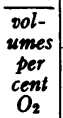 & $\begin{array}{c}\text { per } \\
\text { cent }\end{array}$ & $\begin{array}{c}\text { per } \\
\text { cent }\end{array}$ & $\begin{array}{c}\text { per } \\
\text { cent }\end{array}$ & & gm. & $\begin{array}{l}\text { mil- } \\
\text { lions }\end{array}$ & $\begin{array}{l}\text { mil- } \\
\text { lions }\end{array}$ & $\begin{array}{c}\text { mil- } \\
\text { lions }\end{array}$ & $\begin{array}{l}\text { per } \\
\text { cent }\end{array}$ & $\begin{array}{l}\text { per } \\
\text { cent }\end{array}$ & $\begin{array}{l}\text { per } \\
\text { cent }\end{array}$ & $\begin{array}{c}\text { per } \\
\text { cent }\end{array}$ & $\begin{array}{l}\text { per } \\
\text { cent }\end{array}$ & $\begin{array}{l}\text { per } \\
\text { cent }\end{array}$ & $\begin{array}{l}\text { per } \\
\text { cent }\end{array}$ & $\begin{array}{l}\text { per cent } \\
\text { of } \\
\text { injected }\end{array}$ & $\begin{array}{c}\text { per cent } \\
\text { of } \\
\text { average } \\
\text { normal }\end{array}$ & $\begin{array}{l}\text { mg. } \\
\text { per } \\
\text { cent }\end{array}$ & $\begin{array}{l}\text { me. } \\
\text { per } \\
\text { hour }\end{array}$ & \begin{tabular}{|c|} 
mg. \\
per \\
cent
\end{tabular} & $\begin{array}{c}\text { mg. per } \\
\text { hour }\end{array}$ \\
\hline 0 & $\begin{array}{l}15.7 \\
16.1 \\
20.1\end{array}$ & $\left|\begin{array}{l}4.46 \\
4.32 \\
1.52\end{array}\right|$ & $\begin{array}{l}3.15 \\
2.54 \\
4.26\end{array}$ & $\begin{array}{l}7.61 \\
6.86 \\
5.78\end{array}$ & \begin{tabular}{|l|}
1.41 \\
1.70 \\
0.36
\end{tabular} & $\left|\begin{array}{l}0.9 \\
1.6 \\
3.1\end{array}\right|$ & $\begin{array}{r}106.00 \\
13.49 \\
7.27\end{array}$ & $\begin{array}{r}50.88 \\
11.48 \\
4.10\end{array}$ & $\begin{array}{l}0.58 \\
1.08 \\
0.26\end{array}$ & $\begin{array}{r}100 \\
83 \\
100\end{array}$ & $\begin{array}{l}0 \\
3 \\
0\end{array}$ & $\begin{array}{l}0 \\
0 \\
0\end{array}$ & $\begin{array}{l}0 \\
0 \\
0\end{array}$ & $\begin{array}{r}0 \\
14 \\
0\end{array}$ & $\begin{array}{l}0 \\
0 \\
0\end{array}$ & $\begin{array}{l}0 \\
0 \\
0\end{array}$ & $\begin{array}{l}60.9 \\
37.0 \\
83.2\end{array}$ & $\begin{array}{l}48.3 \\
33.5 \\
74.7\end{array}$ & $\begin{array}{r}117 \\
94 \\
125\end{array}$ & $\begin{array}{r}0.0 \\
12.0 \\
5.0\end{array}$ & $\begin{array}{l}243 \\
245 \\
187\end{array}$ & $\begin{array}{r}2.0 \\
97.0 \\
8.0\end{array}$ \\
\hline 1 & 17.0 & 3.06 & 2.08 & 5.14 & 1.47 & 2.5 & 11.02 & 3.39 & 1.75 & 100 & 0 & 0 & 0 & 0 & 0 & 0 & 54.8 & 35.2 & 109 & 22.0 & 184 & 50.0 \\
\hline 0 & 16.5 & 2.29 & 2.60 & 4.89 & 0.88 & 5.4 & 9.20 & 27.00 & 13.95 & 16 & 0 & $\mathbf{0}$ & 28 & 64 & 0 & 0 & 19.8 & 19.8 & 109 & 31.0 & 192 & 86.0 \\
\hline & $\begin{array}{l}15.0 \\
14.2\end{array}$ & $\begin{array}{l}1.23 \\
3.68\end{array}$ & $\begin{array}{l}2.68 \\
2.26\end{array}$ & $\begin{array}{l}3.91 \\
5.94\end{array}$ & $\begin{array}{l}0.46 \\
1.63\end{array}$ & \begin{tabular}{|l|}
2.9 \\
3.3
\end{tabular} & $\begin{array}{l}25.46 \\
39.44\end{array}$ & $\begin{array}{l}26.80 \\
23.20\end{array}$ & $\begin{array}{r}12.46 \\
4.06 \\
\end{array}$ & $\begin{array}{r}90 \\
0\end{array}$ & $\begin{array}{l}0 \\
0\end{array}$ & $\begin{array}{l}8 \\
0\end{array}$ & $\begin{array}{l}0 \\
0\end{array}$ & $\begin{array}{l}2 \\
0\end{array}$ & $\begin{array}{l}0 \\
0\end{array}$ & 0 & $\begin{array}{l}56.4 \\
15.6\end{array}$ & $\begin{array}{l}46.3 \\
15.9\end{array}$ & $\begin{array}{l}104 \\
115\end{array}$ & $\begin{array}{r}0.0 \\
32.0\end{array}$ & \begin{tabular}{|l|}
186 \\
218
\end{tabular} & $\begin{array}{r}8.0 \\
89.0\end{array}$ \\
\hline 8 & 7.7 & 3.31 & 2.03 & 5.34 & 1.63 & 3.6 & 36.48 & 5.93 & 0.52 & 0 & 0 & 0 & 0 & 0 & 0 & 100 & $\begin{array}{r}10.0 \\
4.9\end{array}$ & $\begin{array}{l}10.9 \\
13.5\end{array}$ & 102 & 46.0 & 243 & 410.0 \\
\hline 9 & $\begin{array}{l}11.5 \\
11.5\end{array}$ & $\begin{array}{l}3.07 \\
3.69\end{array}$ & $\begin{array}{l}2.54 \\
2.44\end{array}$ & $\begin{array}{l}5.61 \\
6.13\end{array}$ & $\begin{array}{l}1.21 \\
1.50\end{array}$ & $\begin{array}{l}4.6 \\
1.9\end{array}$ & $\begin{array}{c}190.68 \\
++\end{array}$ & $\begin{array}{c}77.20 \\
+\end{array}$ & $\begin{array}{c}4.48 \\
+\end{array}$ & $\begin{array}{l}67 \\
0\end{array}$ & $\begin{array}{r}13 \\
0\end{array}$ & $\begin{array}{l}0 \\
0\end{array}$ & $\begin{array}{l}0 \\
0\end{array}$ & $\begin{array}{r}12 \\
0\end{array}$ & $\begin{array}{l}0 \\
0\end{array}$ & $\begin{array}{r}8 \\
100\end{array}$ & $\begin{array}{r}14.1 \\
8.0\end{array}$ & $\begin{array}{r}18.7 \\
6.3\end{array}$ & $\begin{array}{l}131 \\
135\end{array}$ & $\begin{array}{r}18.0 \\
4.7\end{array}$ & \begin{tabular}{|l|}
221 \\
261
\end{tabular} & $\begin{array}{r}52.0 \\
122.0\end{array}$ \\
\hline 1 & 16.5 & 0.71 & 3.35 & 4.06 & 0.21 & 5.4 & 0.24 & 12.40 & 4.96 & 33 & 0 & 2 & 0 & 34 & 8 & 이 & 56.7 & 3 & 121 & 14.0 & 210 & 62.0 \\
\hline 9 & 10.1 & 1.34 & 3.47 & 4.81 & 0.39 & 6.5 & .31 & 6.82 & 1.86 & 70 & 0 & 20 & 10 & 0 & 0 & 0 & 67.8 & 144.0 & 134 & 25.0 & 169 & 20.0 \\
\hline 9 & 17.1 & 1.92 & 2.72 & 4.64 & 0.70 & 7.5 & 0.33 & 24.48 & 5.30 & 80 & 0 & 16 & 4 & 0 & 0 & 0 & 54.6 & 55.9 & 111 & 287.0 & 188 & 410.0 \\
\hline 0 & 13.0 & 1.65 & 2.32 & 3.97 & 0.71 & 3.8 & 0.06 & 14.11 & \begin{tabular}{|l|}
0.88 \\
\end{tabular} & 65 & 0 & 35 & o & 0 & 0 & 0 & 50.1 & 45.8 & 135 & 12.0 & 202 & 16.0 \\
\hline 2 & $\begin{array}{l}19.9 \\
10.5\end{array}$ & $\mid \begin{array}{l}1.29 \\
1.96\end{array}$ & $\begin{array}{l}3.12 \\
2.90\end{array}$ & $\mid \begin{array}{l}4.41 \\
4.86\end{array}$ & $\begin{array}{l}0.41 \\
0.68\end{array}$ & $\begin{array}{l}3.9 \\
3.7\end{array}$ & $\begin{array}{l}0.40 \\
0.39\end{array}$ & $\begin{array}{r}4.29 \\
38.53\end{array}$ & $\begin{array}{l}1.48 \\
7.57\end{array}$ & 30 & 0 & $\begin{array}{r}0 \\
10\end{array}$ & $\begin{array}{r}0 \\
10\end{array}$ & $\begin{array}{l}43 \\
25\end{array}$ & 0 & 27 & 10.2 & 22.2 & 143 & 550.0 & 205 & 628.0 \\
\hline & 19.0 & 4.32 & 3.05 & 7.37 & $\mid 1.42$ & 0.1 & 0.58 & 0.43 & 0.14 & 100 & 0 & 0 & 0 & 0 & 0 & 0 & 50.0 & 78.2 & 117 & 1.0 & 157 & 1.0 \\
\hline & 20.0 & 4.46 & 2.81 & 7.27 & 1.59 & 0.3 & 0.58 & 0.32 & 0.32 & 100 & 0 & 0 & 0 & 0 & 0 & 0 & 74.0 & 88.5 & 112 & 0.0 & 191 & 52.0 \\
\hline & 15.7 & 3.21 & 2.79 & 6.00 & 1.15 & 0.8 & 3.04 & 0.23 & 0.08 & 50 & 0 & 0 & 0 & 50 & 0 & 0 & 28.9 & 11.1 & 118 & 9.0 & 201 & 12.0 \\
\hline
\end{tabular}




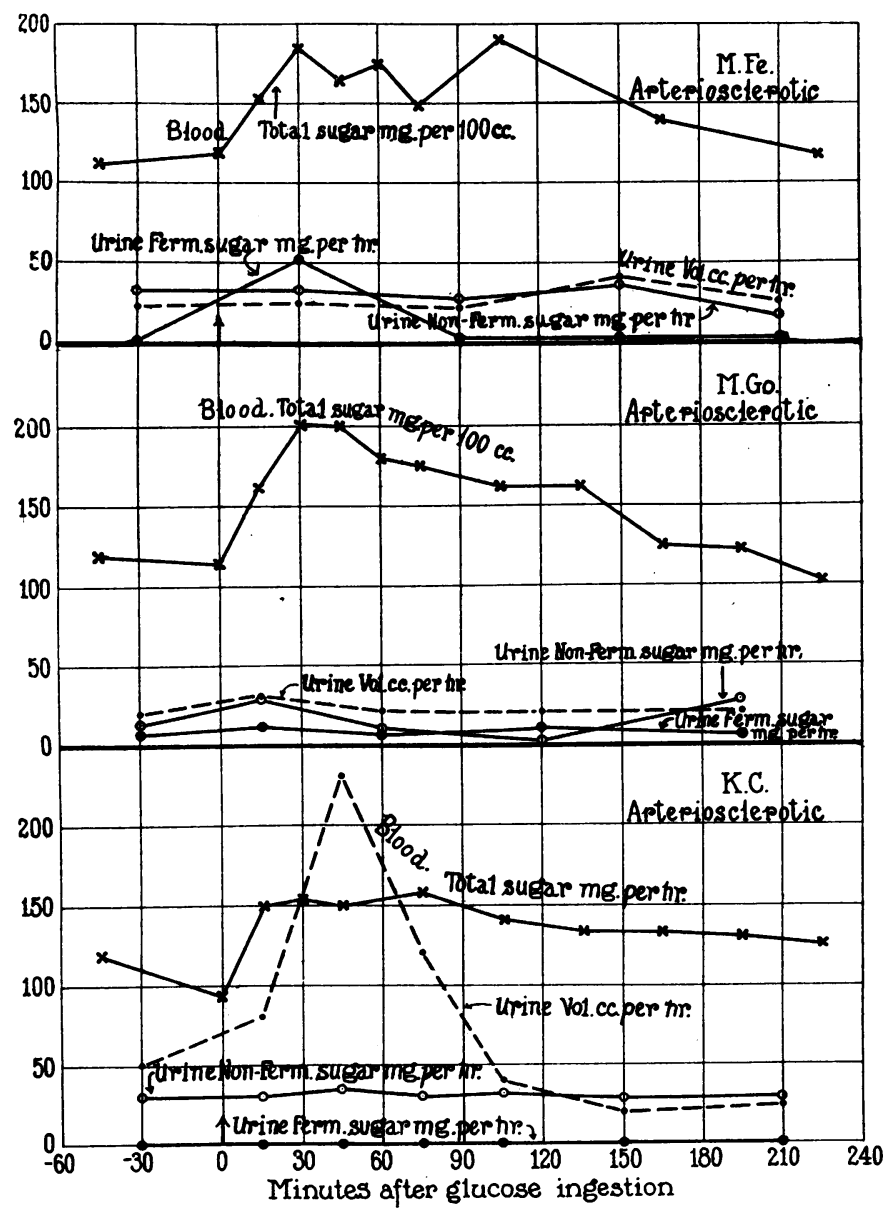

Fig. 11. Data on Subjects with Arteriosclerotic Hemorrhagic NEPHRITIS 


\section{SUMMARY}

Excretion of fermentable sugar has been studied, together with blood sugar curves after glucose ingestion, in normal subjects and in patients with hemorrhagic, degenerative, and arteriosclerotic Bright's disease.

Excretion of fermentable sugar exceeding that observed in the normal control subjects was found to be frequent in all types of the disease. In the degenerative and advanced hemorrhagic types it occurred in all the cases we examined. In half the degenerative cases it amounted to gross glycosuria, the urine containing more than 0.3 per cent of fermentable sugar during fasting and more than 1.0 per cent after glucose ingestion.

The blood sugar values observed before and after glucose ingestion indicated that in all our cases the glycosuria was renal rather than diabetic.

\section{BIBLIOGRAPHY}

Addis, T., J. Am. Med. Assoc., 1925, lxxxv, 163. A Clinical Classification of Bright's Disease.

Eagle, H. S., J. Biol. Chem., 1926, lxxi, 481. On the Nature of the Urine Sugars. Hawkins, J. A., MacKay, E. M., and Van Slyke, D. D., J. Biol. Chem., 1928, lxxviii, p. xxiii. Glucose in the Urine of Normal and Nephritic Subjects.

Linder, G. C., Hiller, A., and Van Slyke, D. D., J. Clin. Invest., 1925, i, 247. Carbohydrate Metabolism in Nephritis.

Lundsgaard, C., and Möller, E., J. Exp. Med., 1922, xxxvi, 559. Investigations of the Oxygen Content of Cutaneous Blood (So Called Capillary Blood).

McIntosh, J. F., Möller, E., and Van Slyke, D. D., J. Clin. Invest., 1928, vi, 467. Studies of Urea Excretion. III. The Influence of Body Size on Urea Output.

Möller, E., McIntosh, J. F., and Van Slyke, D. D., J. Clin. Invest., 1928, vi, 427, 485. Studies of Urea Excretion.' II. Relationship Between Urine Volume and the Rate of Urea Excretion of Normal Adults. IV. Relationship Between Urine Volume and Rate of Urea Excretion by Patients with Bright's Disease.

Neubauer, E., Biochem. Z., 1910, xxv, 284. Über Hyperglykämie bei Hochdrucknephritis und die Beziehung zwischen Glykämie and Glucosurie beim Diabetes mellitus.

Van Slyke, D. D., and Hawkins, J. A., J. Biol. Chem., 1928, lxxix, 739. A Gasometric Method for Determination of Reducing Sugars and its Application to Analysis of Blood and Urine.

Van Slyke, D. D., and Hawkins, J. A., J. Biol. Chem., 1929, lxxxiii, 51. Gasometric Determination of Fermentable Sugar in Blood and Urine. 